04

\title{
Параметры пучковой плазмы, формируемой форвакуумным плазменным источником ленточного электронного пучка в системе транспортировки без магнитного поля
}

\author{
(C) А.С. Климов, ${ }^{1}$ М.И. Ломаев, ${ }^{2,3}$ Е.М. Окс, ${ }^{1,2}$ А.П. Андрейчик ${ }^{1}$ \\ ${ }^{1}$ Томский государственный университет систем управления и радиоэлектроники, \\ 634050 Томск, Россия \\ ${ }^{2}$ Институт сильноточной электроники СО РАН, \\ 634055 Томск, Россия \\ ${ }^{3}$ Национальный исследовательский Томский государственный университет, \\ 634050 Томск, Россия \\ e-mail: klimov@main.tusur.ru
}

(Поступило в Редакцию 29 марта 2016 г.)

\begin{abstract}
Проведены исследования процессов образования пучковой плазмы, формируемой форвакуумным плазменным источником ленточного электронного пучка в условиях его транспортировки без сопровождающего магнитного поля. Определены условия зажигания в области транспортировки пучка пучково-плазменного разряда, позволяющего формировать плазменное образование типа „плазменного листа“ с концентрацией порядка $\sim 10^{16} \mathrm{~m}^{-3}$ и температурой электронов $1-2.5 \mathrm{eV}$. Достигнутые значения параметров и размеры плазмы позволяют использовать ее в технологиях поверхностной модификации плоских протяженных изделий.
\end{abstract}

DOI: 10.21883/JTF.2017.02.44124.1825

\section{Введение}

Пучковая плазма, образованная при взаимодействии ускоренного электронного пучка с остаточным газом, представляет интерес для плазмохимических процессов [1], травления и очистки поверхности различных материалов, синтеза защитных и функциональных покрытий $[2,3]$, стерилизации внутренних поверхностей полых изделий [4], а также для ряда других применений. Оптимальная область давлений для формирования пучковой плазмы лежит в пределах $1-40 \mathrm{~Pa}$ [5].

Форвакуумные плазменные источники электронов [6] представляют собой одно из направлений современного развития источников электронов с плазменным катодом [7]. Они обеспечивают возможность получения электронных пучков непосредственно в области повышенных давлений форвакуумного диапазона, близких к оптимальным для синтеза пучковой плазмы. Использование ленточного электронного пучка позволяет создавать пучковую плазму типа „плазменного листа“ с площадью в сотни квадратных сантиметров [8]. Результаты исследований параметров и характеристик пучковой плазмы, формируемой форвакуумным плазменным источником ленточного электронного пучка, представлены нами ранее в [9]. В этих экспериментах электронный пучок транспортировался в слабом продольном магнитном поле. Для целого ряда применений присутствие магнитного поля нежелательно или даже неприемлемо. Кроме этого, возможное проникновение рассеянного магнитного поля из области транспортировки в ускоряющий промежуток может оказать влияние на снижение его электрической прочности.
Отмеченные обстоятельства стимулировали постановку специальных исследований, направленных на изучение особенностей формирования протяженных плазменных образований, генерируемых форвакуумным плазменным источником ленточного электронного пучка в системе транспортировки пучка без магнитного поля. Результаты проведенных исследований представлены в настоящей работе.

\section{Техника и методика эксперимента}

Эксперименты проводились на вакуумной установке ЭЛУ-1А, состоящей из прямоугольной вакуумной камеры размерами $0.4 \times 0.7 \times 0.4 \mathrm{~m} \mathrm{c}$ механической системой откачки (рис. 1). Для формирования непрерывного ленточного электронного пучка использовался форвакуумный плазменный электронный источник на основе разряда с полым катодом [10]. Полый катод разрядной ячейки электронного источника 1 представлял собой открытый с нижнего торца прямоугольный параллелепипед сечением $120 \times 75 \mathrm{~mm}$ и глубиной $40 \mathrm{~mm}$. Плоский анод 2 был удален от катода 1 на расстояние $5 \mathrm{~mm}$. В аноде было выполнено эмиссионное окно размером $100 \times 10 \mathrm{~mm}$, перекрытое мелкоструктурной вольфрамовой сеткой 3 . Размер элементарной ячейки сетки $0.6 \times 0.6 \mathrm{~mm}$, геометрическая прозрачность $-80 \%$. Извлечение электронов из разрядной плазмы и формирование ленточного электронного пучка с начальным прямоугольным поперечным сечением $100 \times 10 \mathrm{~mm}^{2}$ осуществлялись путем подачи ускоряющего напряжения между анодом 2 и экстрактором 3. Традиционно для 


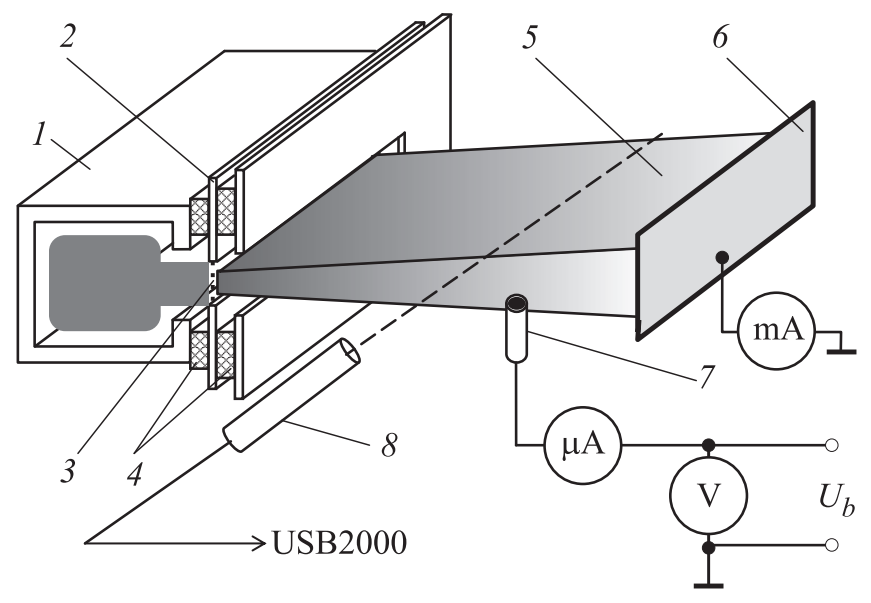

Рис. 1. Схема экспериментальной установки: 1 - протяженный полый катод, 2 - анод, 3 - экстрактор, 4 - изоляторы, 5 - электронный пучок, 6 - коллектор, 7 - одиночный зонд, 8 - спектрометр.

форвакуумных плазменных источников электронов [11] для ускорения электронов в исследуемой области повышенных давлений конструкция ускоряющего промежутка была выполнена таким образом, чтобы предотвратить зажигание в нем разряда по так называемым „длинным путям“. Для электрической изоляции электродов электронного источника использовались капролоновые изоляторы 4.

В экспериментах при неизменном ускоряющем напряжении $2 \mathrm{kV}$ ток пучка варьировал от 100 до $500 \mathrm{~mA}$. Формируемый плазменным источником электронный пучок 5 транспортировался на расстояние $50 \mathrm{~cm}$ и затем принимался на коллектор 6. Для измерения концентрации электронов пучковой плазмы использовался одиночный плоский зонд Ленгмюра 7 с охранным кольцом. Собирающая поверхность зонда представляла собой открытую область медного диска диаметром $4 \mathrm{~mm}$. Дополнительно для защиты от попадания электронов пучка эта область закрывалась металлическим экраном. Зонд располагался в области транспортировки электронного пучка на расстоянии $20 \mathrm{~cm}$ от экстрактора. Определение параметров плазмы осуществлялось по стандартной методике анализа вольт-амперной характеристики зонда [12]. Для исследования свечения плазмы использовался спектрометр „Ocean Optics USB2000“ (диапазон спектральной чувствительности 200-800 nm, полуширина аппаратной функции $\sim 0.3 \mathrm{~nm}$ ). Измерение функции распределения электронов пучка по энергиям осуществлялось методом задерживающего потенциала с помощью специального коллектора, располагаемого на устройстве перемещения. Таким образом, имелась возможность определения функции распределения электронов в зависимости от расстояния до электронного источника.

Давление остаточной атмосферы в вакуумной камере поддерживалось на уровне 5-10 Ра с помощью механи- ческого форвакуумного насоса BocEdwards 80. Состав остаточного газа в камере контролировался квадрупольным масс-анализатором RGA-100.

\section{Результаты экспериментов}

Исследования проводились в атмосфере остаточного газа, содержащей, как показали измерения (рис. 2), молекулы компонентного состава воздуха, а также воды и углеводородов.

При относительно малых токах (150-200 mA) распространение электронного пучка в области транспортировки характеризуется равномерным фиолетовым свечением пучковой плазмы. В спектре излучения плазмы при данных условиях наиболее интенсивны полосы первой отрицательной $(1-)$ системы азота с длинами волн 391.4 и $427.8 \mathrm{~nm}$, переход $\left(B^{2} \Sigma_{u}^{+}\right) \rightarrow\left(X^{2} \Sigma_{g}^{+}\right)$(рис. 3, $c$ ). Вероятно, это является следствием того, что для энергии электронов пучка, равной в эксперименте $2 \mathrm{keV}$, сечение ионизации молекул азота прямым электронным ударом из основного состояния молекулы с заселением уровня $B^{2} \Sigma_{u}^{+}$молекулярного иона азота $\sigma_{i}$ существенно превышает сечение возбуждения состояния $C^{3} \Pi_{u}$ молекулы азота прямым электронным ударом из основного состояния молекулы $\sigma_{\text {exc }}[13]$. Поэтому константа скорости ионизации $k_{i}$ молекул азота и соответственно скорость ионизации ударом электронами пучка из основного состояния молекулы значительно больше константы скорости $k_{\text {ехс }}$ и скорости возбуждения молекул азота в том же процессе:

$$
\begin{gathered}
k_{i}=\int \sigma_{i}(v) v f(v) d v \approx \sigma_{i}\left(v_{b}\right) v_{b}, \\
k_{\mathrm{exc}}=\int \sigma_{\mathrm{exc}}(v) v f(v) d v \approx \sigma_{\mathrm{exc}}\left(v_{b}\right) v_{b} .
\end{gathered}
$$

В (1) $f(v), v, v_{b}$ - функция распределения электронов пучка по скоростям, скорость электронов и электронов

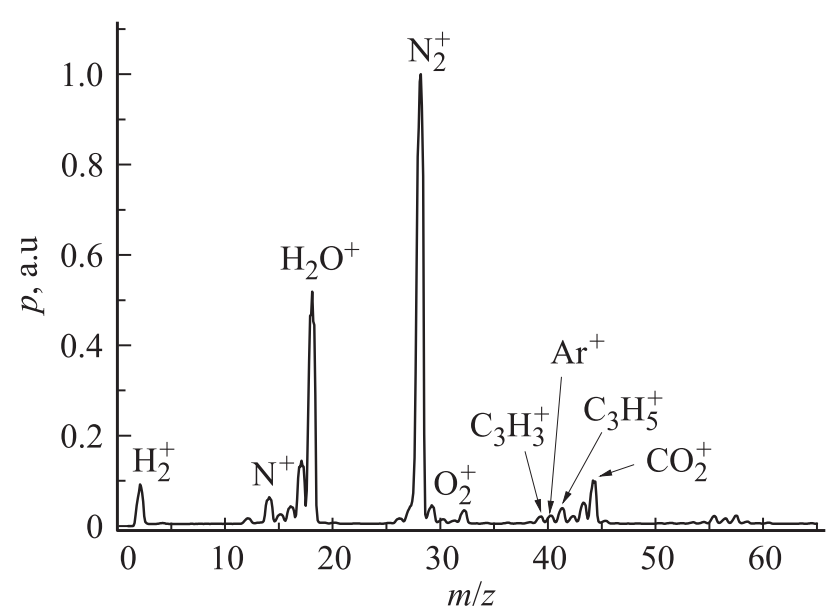

Рис. 2. Состав остаточной атмосферы в области транспортировки электронного пучка. 


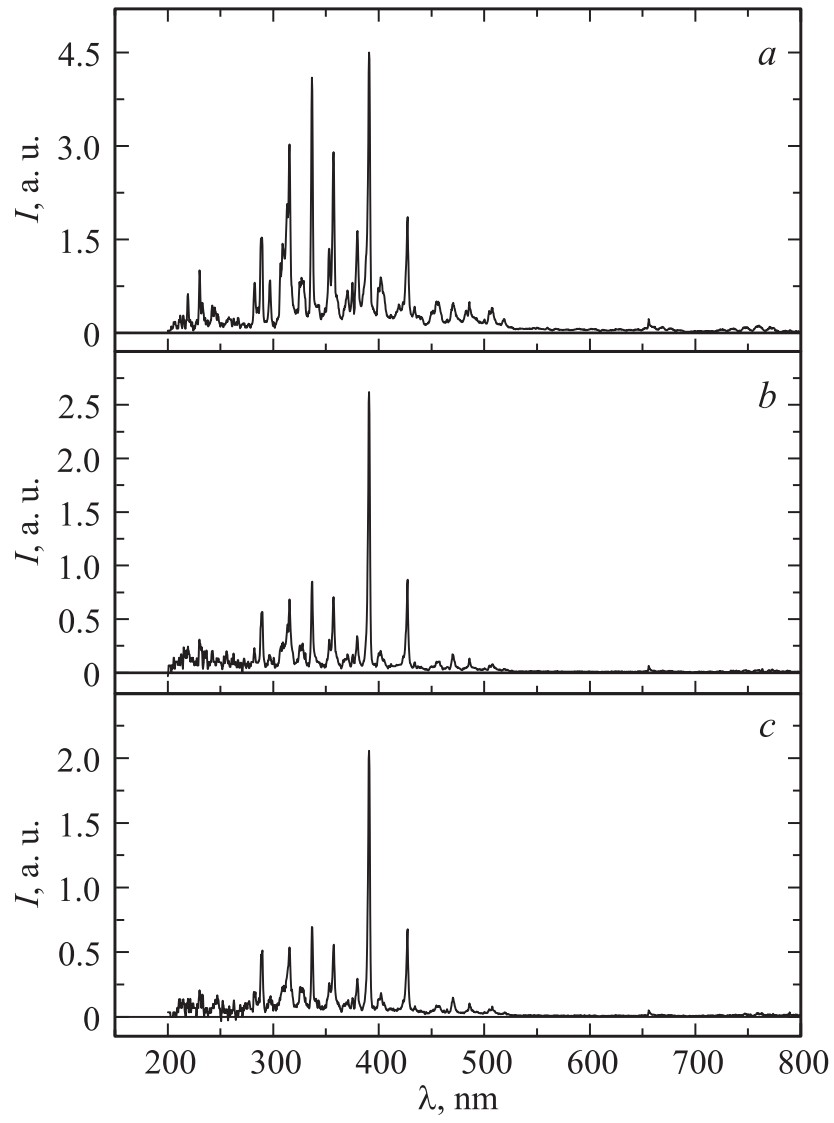

Рис. 3. Спектры излучения плазмы. Ток пучка: $a-400, b-$ 200, c- $160 \mathrm{~mA}$. Давление 7 Ра.

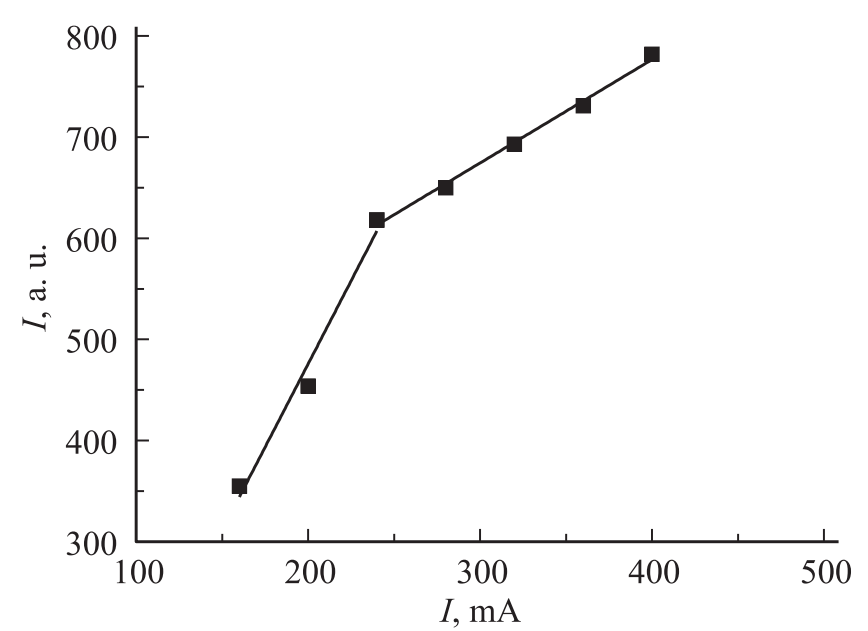

Рис. 4. Зависимость интенсивности свечения линии $391 \mathrm{~nm}$ от тока пучка, давление $7 \mathrm{~Pa}$.

пучка соответственно. В спектре излучения наблюдаются также слабые полосы первой положительной $(1+)$ и второй положительной $(2+)$ систем азота, переходы $B^{3} \Pi_{g} \rightarrow A^{3} \Sigma_{u}^{+}, C^{3} \Pi_{u} \rightarrow B^{3} \Pi_{g}$ соответственно [14].

При превышении током пучка некоторого порогового значения при неизменном давлении на расстоянии порядка $10 \mathrm{~cm}$ от экстрактора фиолетовое свечение пучковой плазмы сменяется ярко-розовым. При этом в спектре излучения плазмы наблюдается рост интенсивности полос $(1+)$ и $(2+)$ систем азота, а также континуума в спектральной области от $\sim 300$ до $\sim 800 \mathrm{~nm}$ (рис. 3, $a, b)$.

Зависимость интенсивности свечения полосы $391.4 \mathrm{~nm}$ от величины тока пучка имеет перегиб и может быть представлена в виде двух линейных участков, отличающихся углом наклона к оси абсцисс (рис. 4).

Интенсивность полос $(2+)$ системы азота с длинами волн 337.1 и $357.7 \mathrm{~nm}$ в свечении плазмы немонотонным образом изменяется в области транспортировки электронного пучка (рис. 5), достигая максимума на некотором оптимальном расстоянии от выходного сечения экстрактора.

Зондовые измерения концентрации и температуры электронов пучковой плазмы (рис. 6) показали, что

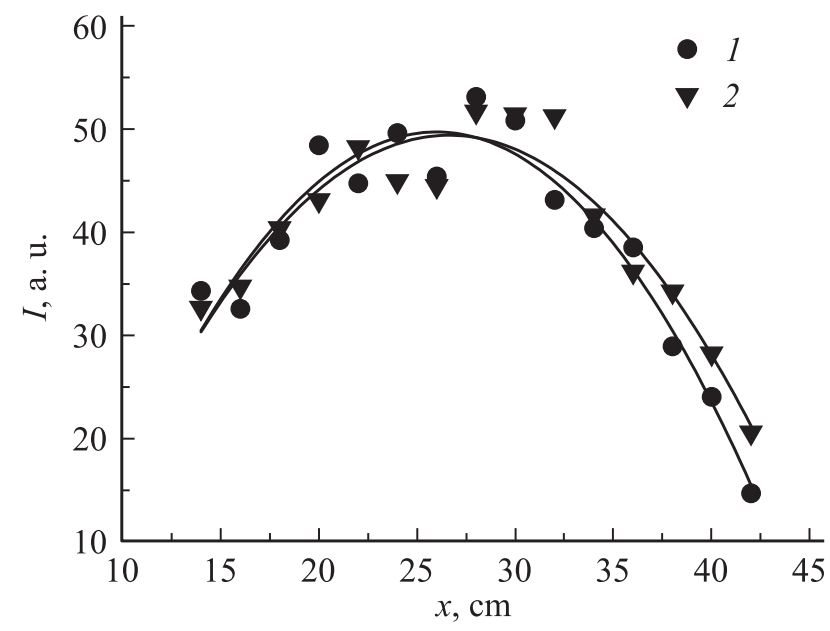

Рис. 5. Зависимость интенсивности свечения пучковой плазмы от расстояния до плоскости инжекции электронного пучка: $1-336,2-357 \mathrm{~nm}$. Ток пучка $200 \mathrm{~mA}$, давление $7 \mathrm{~Pa}$.

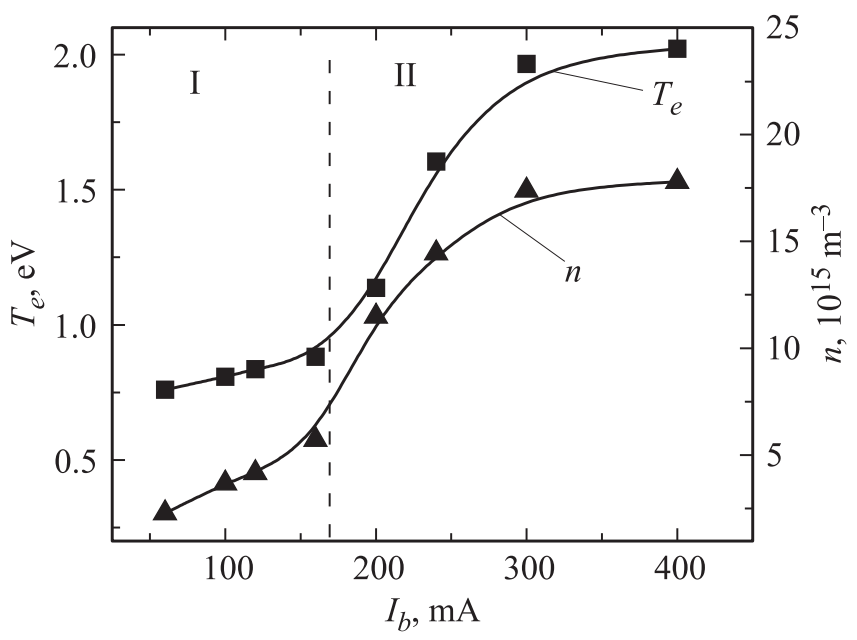

Рис. 6. Зависимость температуры электронов 1 и концентрации пучковой плазмы 2 от тока пучка. Давление $7 \mathrm{~Pa}$. 


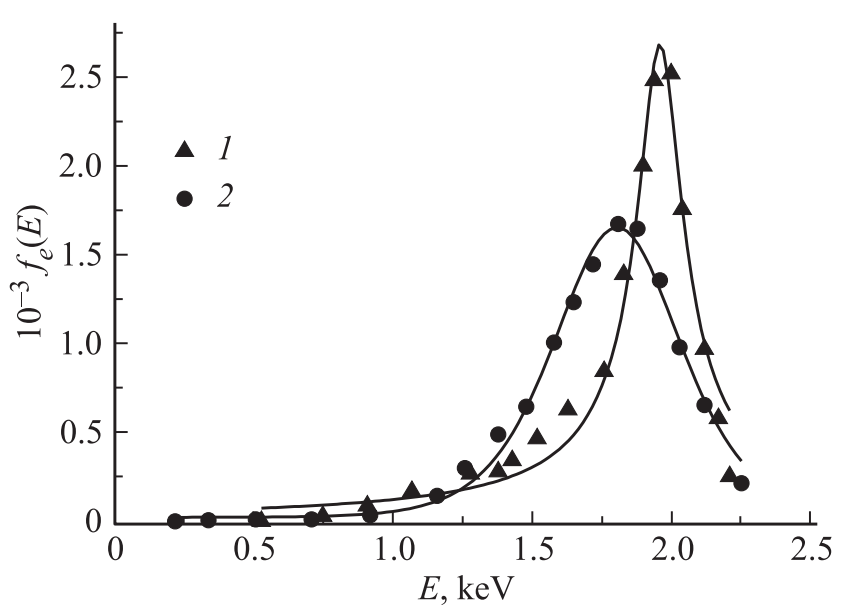

Pис. 7. Функция распределения электронов по энергиям для различных расстояний от экстрактора электронного источника: $1-20,2-45 \mathrm{~cm}$. Ток пучка $200 \mathrm{~mA}$, давление $7 \mathrm{~Pa}$.

относительно слабый рост концентрации и температуры электронов плазмы при токах пучка $50-180 \mathrm{~mA}$ (рис. 6 , область I) сменяется резким ростом при превышении током пучка значений $180-200 \mathrm{~mA}$ (рис. 6, область II).

Функция распределения ускоренных электронов по энергиям, измеренная в области транспортировки пучка для различных расстояний от экстрактора, приведена на рис. 7. Видно, что по мере распространения электронного пучка энергетический спектр электронов пучка заметно деформируется.

Варьирование давления газа в указанных пределах принципиально не изменяло полученных экспериментальных зависимостей, но влияло на пороговые значения тока пучка, при которых наблюдались характерные переходы на этих зависимостях. При этом пороговое значение тока изменялось пропорционально давлению. Аналогичная тенденция наблюдалась при относительно небольших изменениях величины ускоряющего напряжения.

\section{Обсуждение}

Результаты проведенных экспериментов указывают на существование условий, при которых достаточно резко изменяется характер взаимодействия электронов пучка и пучковой плазмы. Соотношение мощности излучения в диапазоне длин волн от 200 до $874 \mathrm{~nm}$ (рис. 3) для токов пучка 400, 200 и $160 \mathrm{~mA}$ составляет $4.1: 1.2: 1$ соответственно, что указывает на качественное изменение параметров плазмы при превышении током пучка значения $200 \mathrm{~mA}$ в условиях постоянства ускоряющего напряжения и давления газа. Пороговый характер таких изменений позволяет предположить возникновение пучковой неустойчивости и, как следствие, зажигание пучково-плазменного разряда [15]. На правомерность такого предположения указывают повышение температуры и концентрации электронов плазмы, а также изменения в спектре излучения пучковой плазмы, которые могут быть связаны с различием в скоростях возбуждения нейтральных молекул и молекулярных ионов азота. Возбуждение излучающих частиц может происходить как в столкновениях с электронами пучка, так и электронами плазмы.

Регистрируемое повышение плотности и температуры электронов плазмы с ростом тока пучка (рис. 6) приводит к существенному повышению скорости возбуждения излучающих частиц электронами плазмы, что объясняет наблюдаемое изменение спектра излучения плазмы.

Известно, что для эффективной передачи энергии электронов пучка плазменным электронам необходимо выполнение условия [15]:

$$
\omega_{p e}\left(\frac{n_{e}}{N_{i}}\right)^{\frac{1}{3}}>5 v_{e n}
$$

где $\omega_{p e}=5 \cdot 10^{4} \sqrt{N_{i}}$ - ленгмюровская электронная частота плазмы,

$$
n_{e}=\frac{j_{e}}{e} \sqrt{\frac{m}{2 e U_{a}}}
$$

- концентрация электронов в пучке $\left(j_{e}-\right.$ плотность тока пучка, $m$ и $e-$ масса и заряд электрона соответственно, $U_{a}$ - ускоряющее напряжение), $N_{i}$ - концентрация плазмы, $v_{e n}=\sigma_{e n} v_{e p} N_{a}$ - частота столкновения плазменных электронов с нейтралами $\left(\sigma_{e n}-\right.$ сечение столкновений электронов с молекулами газа, $v_{e p}-$ скорость электронов плазмы).

Проведенные оценки показывают, что в нашем случае при плотности тока пучка $j_{b}=0.1-0.5 \mathrm{~mA} / \mathrm{mm}^{2}$, ускоряющем напряжении $U_{a}=2 \mathrm{kV}$, концентрации пучковой плазмы $N_{i}=(0.2-2) \cdot 10^{16} \mathrm{~m}^{-3}$ и давлении $p=7 \mathrm{~Pa}$, имеющих место в представленных экспериментах, соотношение (1) оказывается справедливым, что косвенно подтверждает наличие пучково-плазменного разряда (ППР) в условиях эксперимента.

Одним из критериев возникновения ППР является заметная деформация энергетического спектра электронов пучка [16]. Длина релаксации энергии пучка может быть найдена с помощью нерелятивистской формулы Бете [17] (в единицах СГС), описывающей скорость потерь энергии пучка при его прохождении сквозь газ:

$$
\frac{d E}{d x}=-2 \pi N_{\mathrm{mol}} Z_{\mathrm{mol}} e^{4} \frac{1}{E_{b}} \ln \frac{E}{E_{0}},
$$

где $E_{b}$ - энергия пучка в $\mathrm{eV}, x$ - расстояние, проходимое пучком в газе, $N_{\mathrm{mol}}-$ концентрация молекул газа, $Z_{\mathrm{mol}}-$ зарядовое число атомов газа, $e-$ заряд электрона, и $E_{0} \sim 100 \mathrm{eV}-$ средняя энергия возбуждения одной молекулы, примерно одинаковая для большинства газов. Выражение (3) верно для $E \gg E_{0}$. Пренебрегая зависимостью от координаты $x$ величины $E$ 
в логарифме путем интегрирования, получаем длину релаксации энергии пучка $R_{b}$ :

$$
R_{b}=\frac{E^{2}}{4 \pi N_{\mathrm{mol}} Z_{\mathrm{mol}} e^{4}}\left(\ln \frac{E}{E_{0}}\right)^{-1} .
$$

При энергии электронов пучка $2 \mathrm{keV}$, давлении газа (азота) $7 \mathrm{~Pa}$ длина релаксации составляет порядка $100 \mathrm{~cm}$, при этом на длине $40 \mathrm{~cm}$ потери энергии, рассчитанные по (4), составляют порядка $50 \%$.

Экспериментальные функции распределения электронов по энергиям (рис. 7), измеренные в области транспортировки электронного пучка на различных расстояниях от экстрактора электронного источника, также указывают на возможность зажигания ППР. Вблизи электронного источника релаксации пучка не происходит, и вид функции распределения для этого случая (рис. 7, кривая 1) свидетельствует о том, что практически все электроны пучка движутся с начальной энергией $\sim 2 \mathrm{keV}$, участвуя только в парных столкновениях. Удаление от плоскости ввода электронного пучка в газ приводит к заметному уширению энергетического спектра (рис. 7, кривая 2), что свидетельствует о частичной релаксации пучка.

На возможность инициирования ППР в отсутствие магнитного поля указывалось в [18]. Однако диапазон давлений, при которых в этих экспериментах наблюдалось зажигание ППР, был существенно ниже.

\section{Заключение}

Результаты проведенных исследований свидетельствуют о возможности зажигания ППР в области транспортировки ленточного пучка, формируемого форвакуумным плазменным источником электронов, даже в отсутствие транспортирующего магнитного поля. В формируемом при этом плазменном образовании типа плазменный лист концентрация плазмы и температура электронов составляют $\sim 10^{16} \mathrm{~m}^{-3}$ и $1-2.5 \mathrm{eV}$ соответственно. Достигнутые значения параметров и размеры плазмы позволяют использовать ее в технологиях поверхностной модификации различных изделий плоских протяженных форм.

Работа выполнена при поддержке РФФИ (№ грант 14-08-00047), а также Минобрнауки (грант № $3.49 .2014 / \mathrm{K})$. Е.М. Окс является исполнителем работы в рамках государственного задания Минобрнауки „Организация и проведение научных исследованийс ${ }^{\text {) }}$ (проект № 783).

\section{Список литературы}

[1] Ивановский Г.Ф., Петров В.И. Ионно-плазменная обработка материалов. М.: Радио и связь, 1986. 232 с.

[2] Walton S., Leonhardt D., Murphy D., Meger R., Fernsler R. // Bull. Am. Phys. Soc. 1999. Vol. 44. N 8. P. 58.
[3] Leonhardt D., Muratore C., Walton S.G. // IEEE T. Plasma Sci. 2005. Vol. 33. N 2. P. 783.

[4] Zolotukhin D., Burdovitsin V., Oks E., Tyunkov A., Yushkov Yu. // J. Phys. Conf. Ser. 2015. Vol. 652. P. 012044.

[5] Fernsler R.F., Manheimer W.M., Meger R.A., Mathew J., Murphy D.P., Pechacek R.E., Gregorc J.A. // Phys. Plasmas. 1998. Vol. 5. N 5. P. 2137.

[6] Бурдовицин В.А., Климов А.С., Медовник А.В., Окс Е.М., Юиков Ю.Г. Форвакуумные плазменные источники электронов. Томск: изд-во Том. ун-та, 2014. 288 с.

[7] Oks E.M. // Plasm. Sourc. Sci. Tech. 1992. Vol. 1. N 4. P. 249-255.

[8] Leonhardt D., Muratore C., Walton S.G., Blackwell D.D., Fernsler R.F., Meger R.A. // Surf. Coat. Tech. 2004. Vol. 177-178. P. 682-687.

[9] Бурдовицин В.А., Окс Е.М., Федоров М.В. // Изв. вузов. Сер. физ. 2004. № 3. С. 74-77.

[10] Бурдовицин В.А., Бурачевский Ю.А., Окс Е.М., Федоров М.В. // Приборы и техника эксперимента. 2003. № 2. C. $127-129$.

[11] Жирков И.С., Федоров М.В., Осипов И.В., Бурдовиuин B.A., Окс E.M. // Приборы и техника эксперимента. 2005. № 6. C. 66-68.

[12] Хаддлстоун Р., Леонард С. Диагностика плазмы. М.: Мир, 1967. $515 \mathrm{c}$.

[13] Pancheshnyi S.V., Starikovskaia S.M., Starikovskii A.Yu. // J. Phys. D: Appl. Phys. 1999. Vol. 32. N 17. P. 2219-2227.

[14] Пирс Р., Гейдон А. Отождествление молекулярных спектров / Пер. с англ. под ред. С.Л. Мандельштама, М.Н. Аланцева. М.: ИЛ, 1949. 240 с.

[15] Иванов А.А., Лейман В.Г. // Физика плазмы. 1977. Т. 3. № 4. C. $780-785$.

[16] Гордиенко С.Г. и др. // Физика плазмы. 1984. Т. 10. № 5. C. $1010-1013$.

[17] Manheimer W.M., Fernsler R.F., Lampe M., Meger R.A. // Plasm. Sourc. Sci. Tech. 2000. Vol. 9. № 3. P. 370.

[18] Попович В.П., Харченко И.Ф., Шустин Е.Г. // Радиотехника и электроника. 1973. Т. 18. № 3. С. 649-651. 